\title{
SENSACIONES TÉRMICAS EN LA PROVINCIA CIENFUEGOS (CUBA)
}

\author{
Claudio Santiago Castillo Oliva \\ Centro Meteorológico Provincial de Cienfuegos, Instituto de Meteorología, Cuba. \\ cscastilloliva90@gmail.com \\ Sinaí Barcia Sardiñas \\ Centro Meteorológico Provincial de Cienfuegos, Instituto de Meteorología, Cuba. \\ sinai@cfg.insmet.cu
}

\section{RESUMEN}

Los estudios referentes a las sensaciones térmicas son de gran interés y utilidad en diferentes sectores de la sociedad, máxime en la provincia Cienfuegos (Cuba) donde existe un alto potencial económico en continuo desarrollo. Es por eso que este trabajo tiene como objetivo caracterizar temporal y espacialmente las sensaciones térmicas en horarios extremos del día en la provincia Cienfuegos durante el período 1981-2010. Para ello se calcularon los índices bioclimáticos Temperatura Efectiva-TE y Temperatura Efectiva Equivalente-TEE los cuales resultan adecuados para evaluar las sensaciones térmicas de los cubanos aclimatados a las condiciones cálidas y húmedas. Como principales resultados se obtuvo que en la provincia, las mañanas de noviembre a abril son generalmente frescas mientras las tardes de ese período pueden ser confortables o calurosas. Esta última situación es común en las mañanas de mayo a octubre cambiando a calurosas o muy calurosas en horas de la tarde. Las mayores diferencias espaciales se encontraron entre el litoral sur oriental y la zona montañosa resaltando esta última por una permanencia de sensaciones frescas o confortables.

Palabras clave: sensación térmica, índices bioclimáticos, temperatura efectiva, temperatura efectiva equivalente, Cienfuegos, Cuba

\section{ABSTRACT}

\section{Thermal Sensations in Cienfuegos Province (Cuba)}

The studies relating to thermal sensations are interesting and useful for different society sectors, especially in Cienfuegos province where a high economic potential in continuous development exist. For that reason this work has the objective to make a temporally and spatially characterization of thermal sensations in extremes hours of a day in Cienfuegos province in the period 1981-2010. Were calculated the bioclimatic index Effective Temperature- TE and Equivalent Effective Temperature- TEE, which fit to evaluate the Cuban people thermal sensations, whom are acclimatized to hot and humid conditions. The principal results shows that in the mornings of November to April are fresh whereas the afternoons are comforting and warming. This last situation is common in the mornings of May to October turning to warming and very warming in the afternoons. The high spatial differences were found between southeastern coastal area and mountain zone, standing out this last one for a permanence of fresh and comforting sensations.

Keywords: thermal sensations, bioclimatic indices, Effective Temperature, Equivalent Effective Temperature, Cienfuegos, Cuba 


\section{INTRODUCCIÓN}

Una manifestación objetiva de la influencia del clima sobre el hombre son las sensaciones térmicas, que se define como aquella sensación aparente percibida por las personas en función de los parámetros determinantes del ambiente en el cual se mueven y que depende de la relación entre el calor que produce el metabolismo del cuerpo y el disipado hacia el entorno (Urriola, 2009). A esto se le suman aquellos factores personales como la edad, el sexo, el nivel de actividad física, la alimentación, la vestimenta, etc., pero sobre todo el nivel de aclimatación que presenta el cuerpo humano ante una condición ambiental específica.

Debido a las aplicaciones que devienen del conocimiento de estas temáticas, diversos autores en el mundo han estudiado el comportamiento espacio- temporal de las sensaciones térmicas utilizando para ello índices bioclimáticos. Dentro de los más empleados se encuentran Índice de Voto de Predicción-PMV e Índice por el Porcentaje de Predicción no Satisfecho-PPD (Fanger, 1972), Índice de Hendrick (Hendrick, 1959), Índice de Calor Sofocante (Lecha, 1988), Wind chill (Siple y Passel, 1945), Temperatura Efectiva (Houghtem y Yaglou, 1923) y Temperatura Efectiva Equivalente (Missenard, 1937).

Ejemplos de lo anterior lo constituye el trabajo realizado por Moreno \& Fernández (2004) donde se relaciona el confort térmico con la distribución espacial de las rentas en la Comunidad de Madrid. Siguiendo la línea de la modelación se encuentra la investigación desarrollada por Royé et al. (2012) en la cual se realiza una aproximación al comportamiento espacial y temporal del estrés térmico en Galicia mediante la aplicación del índice bioclimático PET (Physiological Equivalent Temperature) y del modelo RayMan. En este estudio se logra sintetizar en un solo mapa las áreas con mayor riesgo de sufrir situaciones de estrés térmico, tanto por frío como por calor.

Los trabajos sobre confort o bienestar térmico en Cuba comenzaron a partir de la década de los '80 del siglo XX, los cuales presentaban un enfoque climático-descriptivo. A partir de los años '90 se desarrollaron diferentes investigaciones que respondían a problemáticas de diferentes sectores de la sociedad, tal es el caso de los autores Osorio et al. (1991) que trabajaron sobre el bienestar turístico de Varadero; o el de Lecha (1993) que resalta la importancia de las sensaciones térmicas en el desarrollo de tratamientos climatoterapéuticos en la provincia Cienfuegos; además de Guevara et al. (1997) que valoraron las características del clima en Cuba con vistas a su aprovechamiento en función de lograr una mejoría en las condiciones bioclimáticas de las edificaciones.

Particularmente, la provincia Cienfuegos si bien ha sido caracterizada tanto bioclimática como climáticamente por Lecha (1993) y Barcia (2012) respectivamente, carece de un estudio detallado respecto al comportamiento espacio-temporal de las sensaciones térmicas. Es por ello que el presente trabajo tiene como objetivo caracterizar temporal y espacialmente las sensaciones térmicas en horarios extremos del día en la provincia Cienfuegos. Los resultados expuestos resultan de gran interés en esferas tan importantes como la salud, el turismo, la planificación de actividades al aire libre, la urbanización, el ahorro de energía eléctrica, entre otras.

\section{MATERIALES Y MÉTODOS}

La provincia Cienfuegos se encuentra situada al centro - sur de la isla de Cuba, entre los $21^{\circ} 22^{\prime}$ y $22^{\circ} 35^{\prime}$ de latitud norte y entre los $80^{\circ} 20^{\prime}$ y $81^{\circ} 10^{\prime}$ de longitud oeste. Se trata de un territorio principalmente llano hacia su parte occidental mientras que el este es montañoso por la presencia del macizo de Guamuhaya cuya máxima altitud es de $1140 \mathrm{~m}$ sobre el nivel medio del mar. El tipo de clima predominante en este territorio, atendiendo a la clasificación de Köppen modificada, es tropical con verano relativamente húmedo en contraste con la zona montañosa donde habitualmente es templado cálido con lluvias todo el año, (Planos et al., 2013).

Para caracterizar las principales zonas físico-geográficas de la provincia desde el punto de vista de las sensaciones térmicas se seleccionaron 6 estaciones meteorológicas pertenecientes a la red del Instituto de Meteorología (INSMET); de las cuales tres caracterizan la zona sur y costera de la provincia (Girón (GR), Cienfuegos (CF) y Trinidad (TR), una la zona montañosa (Topes de Collantes, TP)) y el resto se corresponden con zonas del interior (Aguada de Pasajeros (AG) y Santo Domingo (SD)) tal y como muestra la Figura 1 y Tabla 1. 
Figura 1. Mapa Físico-Político de la provincia Cienfuegos

(incluye ubicación de las estaciones meteorológicas utilizadas en el estudio)

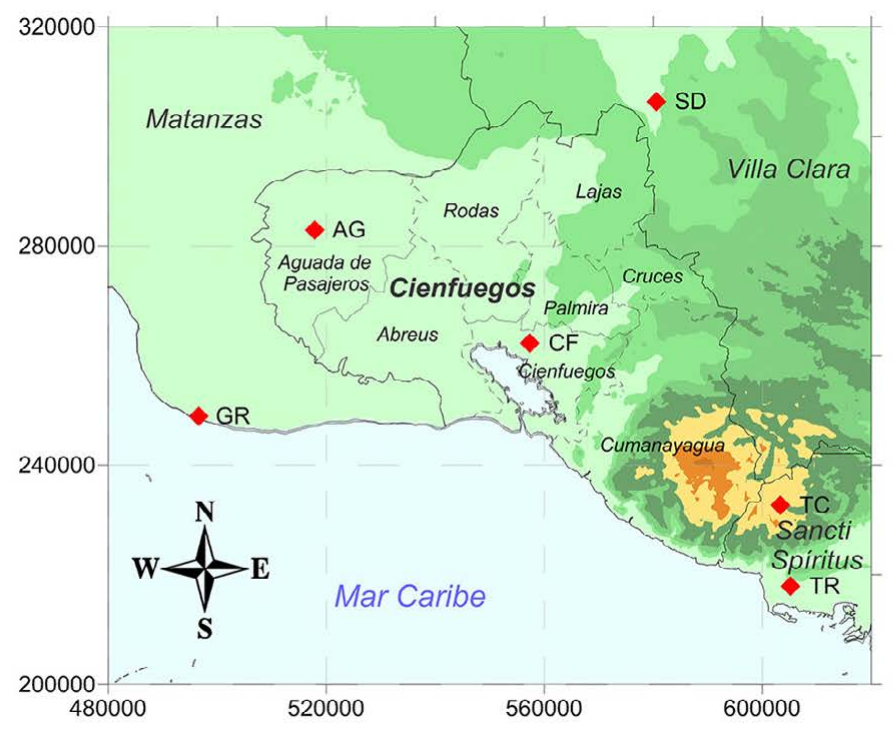

\section{LEYENDA}

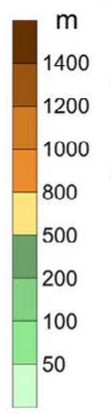

Limites

interprovinciales

Límites

intermunicipales

Estaciones

Meteorológicas

Elaboración propia.

Tabla 1. Estaciones meteorológicas utilizadas en el estudio y parámetros climáticos representativos.

\begin{tabular}{|c|c|c|c|c|c|c|c|}
\hline \multirow[t]{2}{*}{ Estación } & \multirow[t]{2}{*}{ Provincia } & \multicolumn{2}{|c|}{$\begin{array}{c}\text { Coordenadas } \\
\text { Geográficas }\end{array}$} & \multirow{2}{*}{$\begin{array}{c}\text { Altura } \\
(\mathrm{m})\end{array}$} & \multirow{2}{*}{$\begin{array}{c}\text { T. } \\
\text { Media* } \\
\left({ }^{\circ} \mathrm{C}\right)\end{array}$} & \multirow{2}{*}{$\begin{array}{c}\mathrm{Hr} \\
\text { media }^{*} \\
(\%)\end{array}$} & \multirow{2}{*}{$\begin{array}{l}\text { Viento } \\
\text { Medio }^{*} \\
(\mathrm{Km} / \mathrm{h})\end{array}$} \\
\hline & & $\mathrm{X}$ & $\mathrm{Y}$ & & & & \\
\hline Cienfuegos (CF) & Cienfuegos & $-80,4$ & 22,2 & 41,92 & 24.7 & 76 & 7.5 \\
\hline Aguada de Pasajeros (AG) & Cienfuegos & $-80,8$ & 22,1 & 27,39 & 24.4 & 78 & 8.5 \\
\hline Topes de Collantes (TP) & Sancti Spíritus & $-80,0$ & 21,9 & 771,2 & 20.6 & 74 & 9.7 \\
\hline Trinidad (TR) & Sancti Spíritus & $-80,0$ & 21,8 & 53,9 & 25.8 & 77 & 7.2 \\
\hline Santo Domingo (SD) & Villa Clara & $-80,2$ & 22,6 & 46,13 & 24.2 & 78 & 7.7 \\
\hline Girón (GR) & Matanzas & $-81,0$ & 22,1 & 6,0 & 24.7 & 80 & 6.8 \\
\hline
\end{tabular}

Fuente: Barcia, S. (2012)

Se tomaron los datos de la temperatura del aire, humedad relativa y velocidad del viento dentro del período 1981-2010, medidos a las 7:00 a.m. y 1:00 p.m., en cada una de las estaciones seleccionadas. Se consideró a priori que ambos horarios son representativos de los momentos más frío y cálido del día, respectivamente. No obstante, este criterio fue comprobado a través del cálculo de la correlación lineal entre los valores de la temperatura a las 7:00 a.m. y la temperatura mínima diaria, y entre las series de temperatura a la 1:00 p.m. y la máxima diaria, obteniéndose un coeficiente mayor al 0,90 en todos los casos, justificando así lo planteado anteriormente.

Se escogieron los índices Temperatura Efectiva (TE) y Temperatura Efectiva Equivalente (TEE), los cuales son índices bioclimáticos empíricos de sensación térmica calculados sobre la base de la temperatura del aire y la humedad relativa incluyendo la velocidad del viento para el caso de la TEE. Ambos resultan válidos para evaluar la sensación térmica en personas bajo actividad física poco intensa (caminar, labores de oficina, entre otras), vestidas con ropa habitual de verano (0,6 clo de aislamiento térmico del vestuario) y que permanecen en el interior de las edificaciones o en exteriores a la sombra, donde no esté presente el efecto del viento según la TE y con la influencia del mismo según la TEE.

Posteriormente se calcularon la TE y la TEE a las 7:00 a.m. y a la 1:00 p.m. para cada día del período seleccionado mediante la fórmula de Brooks (Bútieva et al. 1984). Sus expresiones completas son: 


$$
\begin{gathered}
T E=t-\frac{G}{80}\left(0,00439 T^{2}+0,456 T+9,5\right) \\
T E E=T E+W[(0,11 T-0,13)-0,002 T G]
\end{gathered}
$$

Donde:

$\boldsymbol{t}$ : Temperatura del aire

$\boldsymbol{G}=\mathbf{1 0 0}-\boldsymbol{r}$, donde r es la humedad relativa del aire en $\%$.

$\boldsymbol{T}=\boldsymbol{t}$ - 37: diferencia entre la temperatura del aire y la del cuerpo humano.

$\boldsymbol{W}$ : velocidad del viento a $2 \mathrm{~m}$ de altura, que proviene de la relación $0,67 \mathrm{~V}$, donde $\mathrm{V}$ es la velocidad del viento a $10 \mathrm{~m}$ de altura (al nivel de la estación meteorológica), en m/s.

Conocidas la TE y la TEE, fueron establecidas las sensaciones térmicas correspondientes a los distintos intervalos (Tabla 2) propuestos por León (1988) que en una primera aproximación pueden considerarse adecuados para la población cubana, aclimatada a las condiciones cálidas y húmedas que imperan durante la mayor parte del año en Cuba. Su análisis temporal se realizó a partir de los gráficos de distribución de frecuencias mensual y anual para determinar la probabilidad de ocurrencia de las sensaciones térmicas en dependencia de la época del año.

Tabla 2. Intervalos de sensación térmica para Cuba, sobre la base de TE y TEE

\begin{tabular}{|c|c|}
\hline Intervalo de TE $/ \mathrm{TEE}$ & Sensación Térmica \\
\hline $\mathrm{TE}-\mathrm{TEE} \leq 12{ }^{\circ} \mathrm{C}$ & Muy fría \\
\hline $12,0<\mathrm{TE}-\mathrm{TEE} \leq 17,0$ & Fría \\
\hline $17,0<\mathrm{TE}-\mathrm{TEE} \leq 22,0$ & Ligeramente fría o fresca \\
\hline $22,0<\mathrm{TE}-\mathrm{TEE} \leq 25,0$ & Confortable \\
\hline $25,0<\mathrm{TE}-\mathrm{TEE} \leq 28,0$ & Calurosa \\
\hline $\mathrm{TE}-\mathrm{TEE}>28,0^{\circ} \mathrm{C}$ & Muy calurosa \\
\hline
\end{tabular}

Fuente: León (1988).

La distribución espacial de las sensaciones térmicas en la provincia Cienfuegos se obtuvo aplicando la metodología de interpolación basada en el análisis de regresión múltiple tomando como partida una rejilla de 4x4 km utilizada ya en otros estudios climáticos y agrometeorológicos en el país (Centella et al. 2006). El objetivo final era predecir el valor de cada índice de sensación térmica en cada punto de rejilla basándose en las principales variables geográficas susceptibles de influir en el comportamiento de la temperatura, humedad y viento y de las cuales se contaba con información en formato de Sistema de Información Geográfica (SIG). Se realizaron análisis de regresión múltiple, Tabla 3, donde se tomaron como variables dependientes los índices de sensación térmica de las estaciones meteorológicas y como variables independientes los factores geográficos como altitud, latitud, longitud y distancia a la costa. Finalmente se utilizaron los coeficientes de regresión, que indicaban el peso que tiene cada variable independiente para reconstruir el índice de sensación térmica en una determinada celda del territorio.

Tabla 3. Ecuaciones de regresión empleadas para el análisis espacial de las sensaciones térmicas.

\begin{tabular}{|c|c|l|c|}
\hline Mes & Horario & \multicolumn{1}{|c|}{ Ecuación de regresión } & $\mathbf{R}^{2}$ \\
\hline \multirow{4}{*}{ Enero } & 7:00 a.m. & $T E=80,55284-2,83886 \boldsymbol{y}-0,00329 \boldsymbol{h}-0,02919 \boldsymbol{d}$ & 0,830 \\
\cline { 2 - 4 } & 1:00 p.m. & $T E=23,75-0,00535 \boldsymbol{h}-0,013 \boldsymbol{d}$ & 0,880 \\
\cline { 2 - 4 } & 7:00 a.m. & $T E E=16,80362-0,00635 \boldsymbol{h}-0,04357 \boldsymbol{d}$ & 0,691 \\
\cline { 2 - 4 } & 1:00 p.m. & $T E E=22,91124-0,00686 \boldsymbol{h}$ & 0,744 \\
\hline \multirow{4}{*}{ Julio } & 7:00 a.m. & $T E=24,03581-0,00396 \boldsymbol{h}$ & 0,901 \\
\cline { 2 - 4 } & 1:00 p.m. & $T E=75,60625+0,33916 \boldsymbol{x}-0,92059 \boldsymbol{y}-0,00486 \boldsymbol{h}+0,00686 \boldsymbol{d}$ & 0,857 \\
\cline { 2 - 4 } & 7:00 a.m. & $T E E=23,60442-0,00583 \boldsymbol{h}-0,00944 \boldsymbol{d}$ & 0,827 \\
\cline { 2 - 4 } & 1:00 p.m. & $T E E=27,51058-0,00514 \boldsymbol{h}$ & 0,652 \\
\hline
\end{tabular}

Donde: $x$ : Longitud, $h$ : Altitud, $y$ : Latitud, $d$ : Distancia a la costa 
Los mapas se confeccionaron utilizando el software libre Quantum GIS 1.8.0, a partir del método de interpolación denominado "inverso de la distancia al cuadrado" y como resultado final se obtuvo ocho capas raster con una resolución espacial de $4 \mathrm{~km}$, cuatro con las temperaturas efectivas medias para los meses de enero y julio en los horarios de las 7:00 a.m. y 1:00 p.m. y cuatro con las temperaturas efectivas equivalentes medias para los mismos meses y horarios, que son los que mejor representan la estacionalidad térmica que caracteriza el clima de la provincia.

\section{CARACTERÍSTICAS DE LAS SENSACIONES TÉRMICAS EN LA PROVINCIA CIENFUEGOS}

\subsection{Análisis temporal de las sensaciones térmicas}

Los índices TE y TEE muestran variaciones diarias y anuales provocadas por las propias variables que ellos agrupan. Por lo que es evidente esperar que ocurran sensaciones térmicas más frecuentes que otras en dependencia de la zona geográfica, el horario y la época del año.

En su conjunto, las sensaciones frescas, frías y muy frías en el horario de las 7:00 a.m. se presentan en los meses de noviembre a abril con una frecuencia superior al $80 \%$ en la mayor parte de la provincia (Figura 2). Esto está condicionado por las temperaturas relativamente bajas, propias de la época del año. Hacia el interior del territorio la probabilidad de las muy frías aumenta alcanzando hasta un $20 \%$ en el mes de enero, sin embargo, en el litoral dichas condiciones se limitan hasta un 10\% como máximo.

Figura 2. Distribución intraanual de la frecuencia (en porcentaje) de las sensaciones térmicas dadas por la TE, 7:00 a.m.

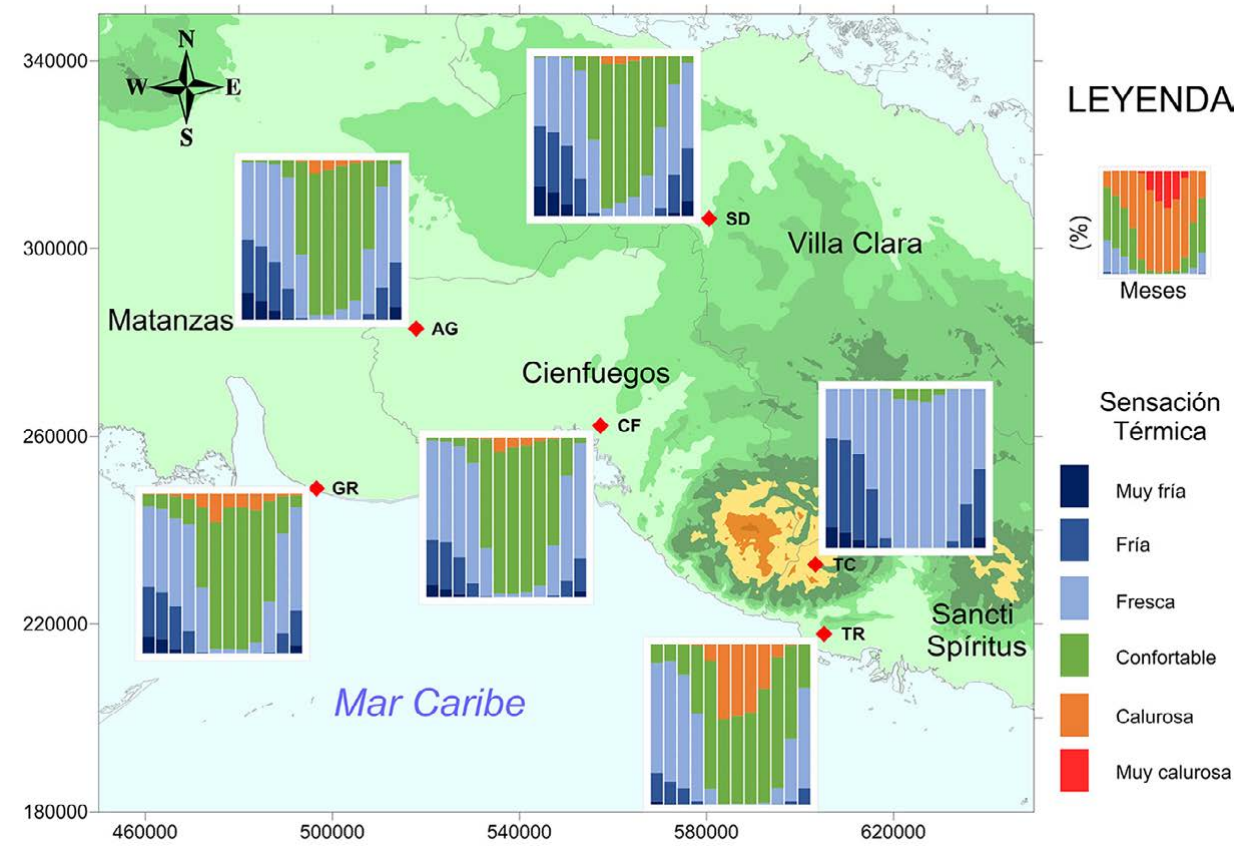

Por otra parte, desde mayo hasta octubre se establecen sensaciones confortables con una frecuencia del 60 al 90\%, excepto en la montaña donde prevalecen condiciones frescas. Es necesario destacar que es poco probable la aparición de sensaciones calurosas en horas de la mañana, no obstante, las mismas son más frecuentes hacia la costa, destacándose el extremo suroriental del territorio, donde pueden ocurrir hasta un $40 \%$ de los días del trimestre junio-agosto, Figura 2.

Aunque en horarios de la mañana la velocidad del viento es mínima en la mayoría de las estaciones meteorológicas escogidas, logra provocar aumentos notables en la frecuencia de las sensaciones extremas por debajo del confort. Como se muestra en la Figura 3, los mayores ascensos se localizan hacia la zona montañosa donde las sensaciones frías conjuntamente con las muy frías pueden ocurrir hasta el $90 \%$ de los días desde diciembre hasta marzo. El aumento de estas condiciones se asocia a la combinación de temperaturas bajas, humedades relativas altas y vientos fuertes, características de este tipo de emplazamiento en el país. 
Figura 3. Distribución intraanual de la frecuencia (en porcentaje) de las sensaciones térmicas dadas por la TEE, 7:00 a.m.

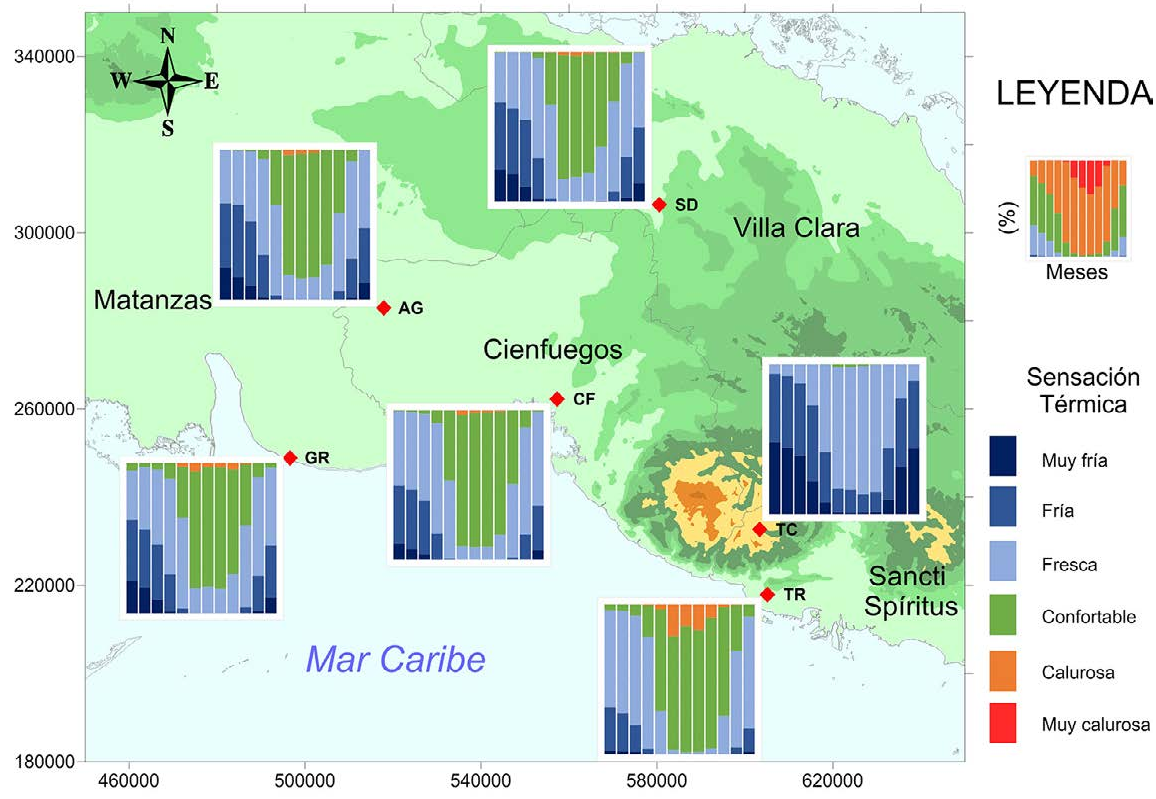

En el horario de la 1:00 p.m. las condiciones cambian considerablemente respecto a lo descrito anteriormente. En la mayor parte del territorio las condiciones de confort sólo están presentes con más del 45\% en el período de noviembre a abril, mientras que la probabilidad restante de esta época se comparte entre la ocurrencia de sensaciones frescas y calurosas. Estas últimas se hacen más frecuentes en los meses estivales donde se da paso a las muy calurosas que llegan a alcanzar un máximo en agosto cercano al 40\%, Figura 4.

Figura 4. Distribución intraanual de la frecuencia (en porcentaje) de las sensaciones térmicas dadas por la TE, 1:00 p.m.

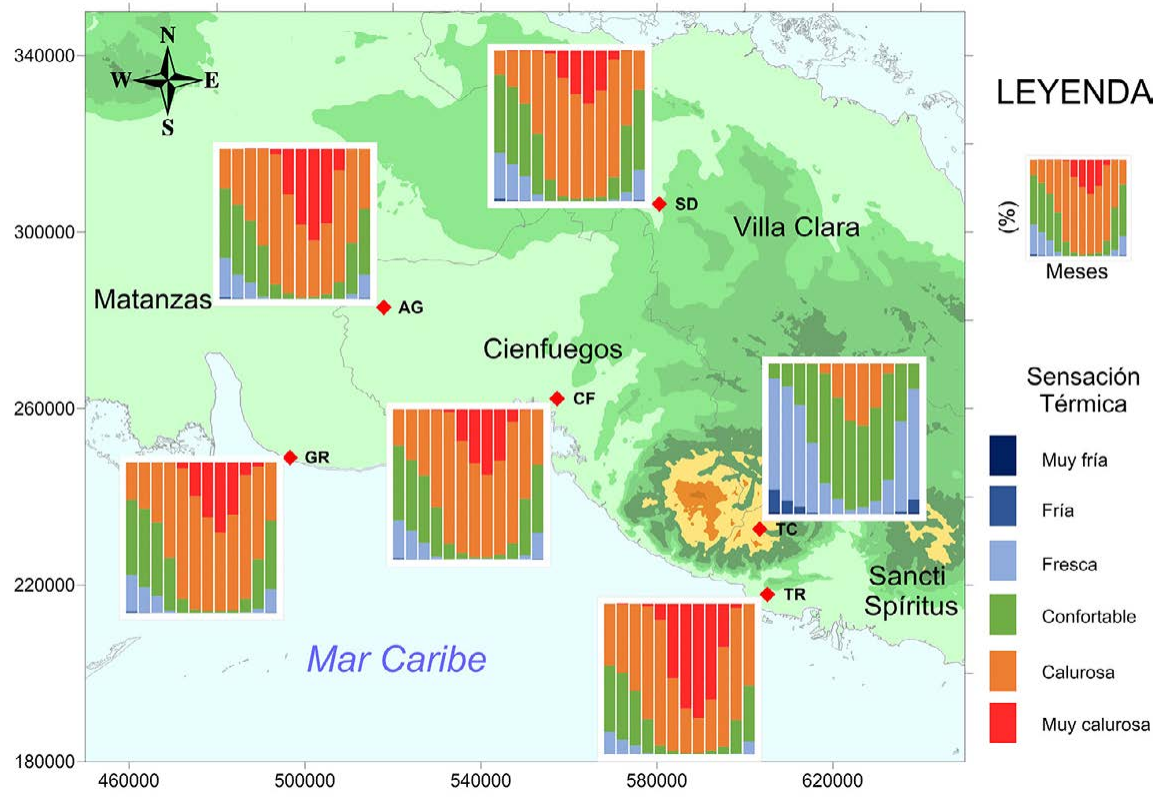

Específicamente, la zona montañosa goza de un escenario favorable desde el punto de vista bioclimático pues las condiciones frescas ocupan más del $50 \%$ de los días de la temporada mayo-septiembre mientras las sensaciones confortables sobrepasan el $50 \%$ en los meses de abril a octubre. La mayor probabilidad de ocurrencia de las condiciones calurosas queda concentrada desde junio a septiembre con un $30 \%$ mostrado por la TE en la Figura 4. Puede observarse también que las condiciones muy calurosas no se presentan en 
esta zona de la provincia, además las sensaciones de disconfort se limitan al verano resaltando la condición de montaña de la estación y la relación inequívoca entre las sensaciones térmicas y la altura.

De igual forma, se hace preciso resaltar que la costa oriental, al situarse a sotavento del sistema montañoso Guamuhaya recibe la mayor carga de radiación solar de la provincia y consecuentemente mantiene temperaturas elevadas en comparación con las demás zonas de estudio. Esto explica por qué en dicha región se encuentran las mayores frecuencias de condiciones de calor extremas. Las sensaciones muy calurosas son habituales desde junio hasta septiembre en más del 50\% de los días llegando a sobrepasar el $70 \%$ en agosto como se expone en la Figura 4.

Al tener en cuenta la influencia del viento en horas de la tarde dado por la TEE puede apreciarse una disminución de las sensaciones muy calurosas en los meses de junio a septiembre. Dicho comportamiento da lugar a un ascenso en la ocurrencia de condiciones calurosas, que aunque provocan malestar o disconfort térmico son menos estresantes para el sistema termorregulador humano que las anteriores. Esto se hace mucho más notable hacia la costa donde son menos frecuentes las calmas y la velocidad media del viento es mayor que hacia las zonas interiores (Figura 5).

Figura 5. Distribución intraanual de la frecuencia (en porcentaje) de las sensaciones térmicas dadas por la TEE, 1:00 p.m.

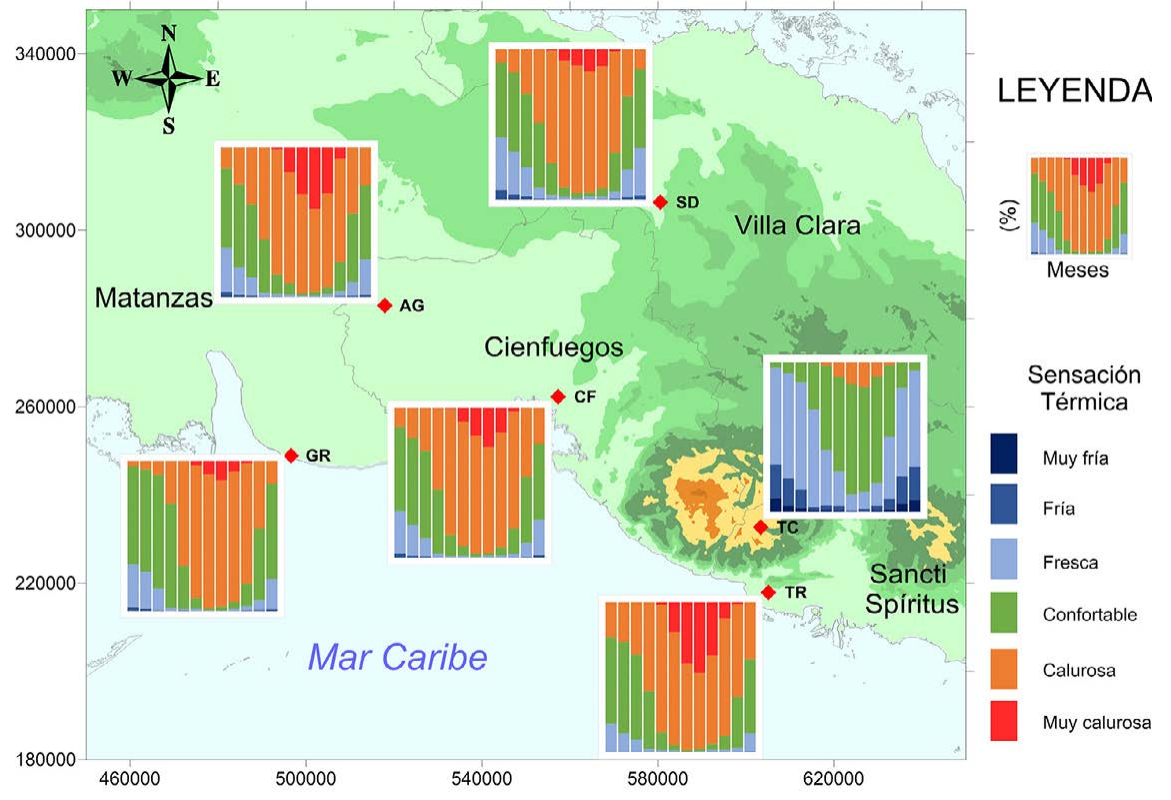

Por su parte, en el período de noviembre a marzo las sensaciones confortables y frescas sufren variaciones positivas haciendo que las calurosas no ocurran en más del 30\%.

\subsection{Análisis espacial de las sensaciones térmicas}

La distribución espacial de las sensaciones térmicas se explica, en general, mediante las condiciones climáticas propias de cada región que imponen una clara estacionalidad anual y por las características físico-geográficas que introducen diferencias significativas entre una zona u otra, ya sea por diferencias altitudinales o por desigual influencia marítima. Los mapas de TE y TEE en meses y horarios contrastantes del año y del día respectivamente, resumen bastante bien estos aspectos y permiten mostrar las características bioclimáticas imperantes en la provincia Cienfuegos.

Como se aprecia en la Figura 6 (izquierda), en el mes de enero a las 7:00 a.m. los menores valores del índice TE se encuentran hacia la montaña y la llanura interior de la provincia haciendo que sobre estas regiones prevalezcan las sensaciones frías que dan lugar a las frescas o ligeramente frías a medida que disminuye la distancia a la costa, coincidente con el sur de los municipios de Abreus y Cumanayagua y la totalidad del municipio Cienfuegos. Al tener en cuenta la influencia del viento (Figura 6, derecha) y aunque el mismo tiene una baja velocidad en este horario, logra imponer sensaciones frías en toda la provincia a excepción de las zonas más altas del sistema montañoso donde predominan las muy frías. 
Figura 6. Sensación térmica del mes de enero a las 7:00 a.m. dadas por la TE (izquierda) y la TEE (derecha). Provincia Cienfuegos, período 1981-2010.
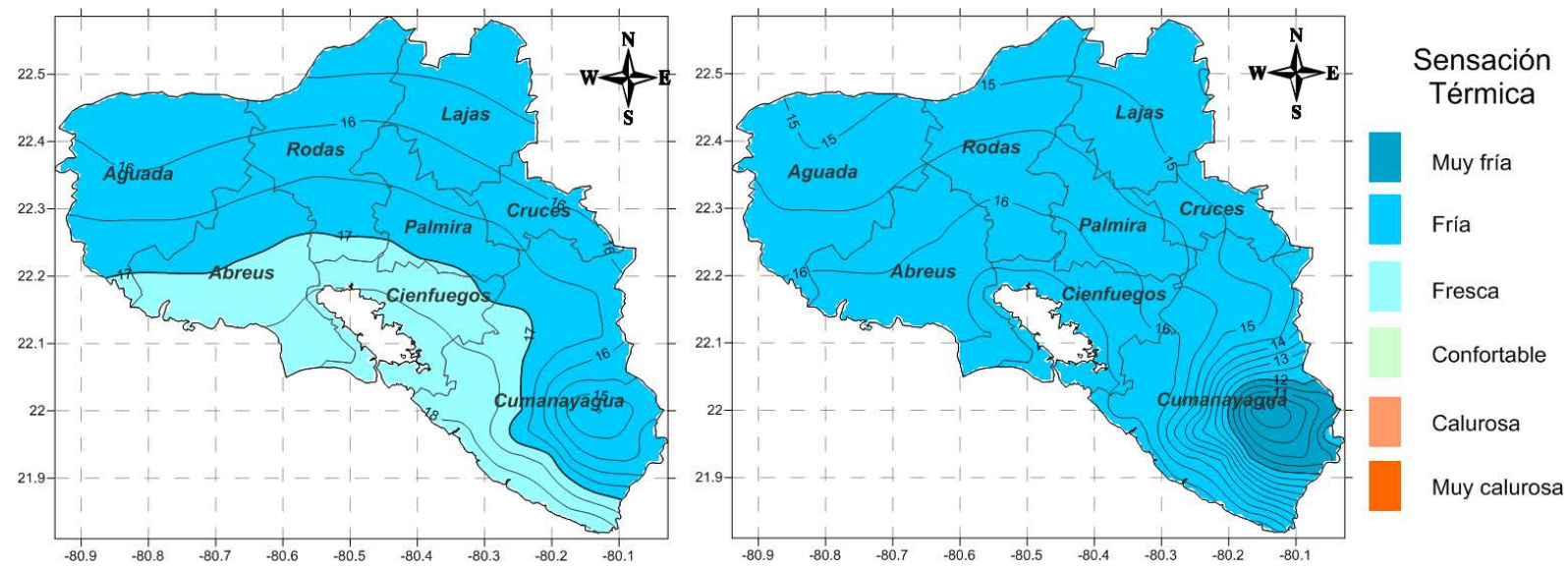

En este mismo mes pero en horas de la tarde prevalecen las condiciones confortables en casi todo el territorio cienfueguero, persistiendo sensaciones ligeramente frías a partir de los $400 \mathrm{~m}$ de altura en la montaña (Figura 7, izquierda) agudizándose hasta sentirse frías en las cumbres más altas debido al efecto del viento (Figura 7, derecha).

Figura 7. Sensación térmica del mes de enero a la 1:00 p.m. dadas por la TE (izquierda) y la TEE (derecha). Provincia Cienfuegos, período 1981-2010.
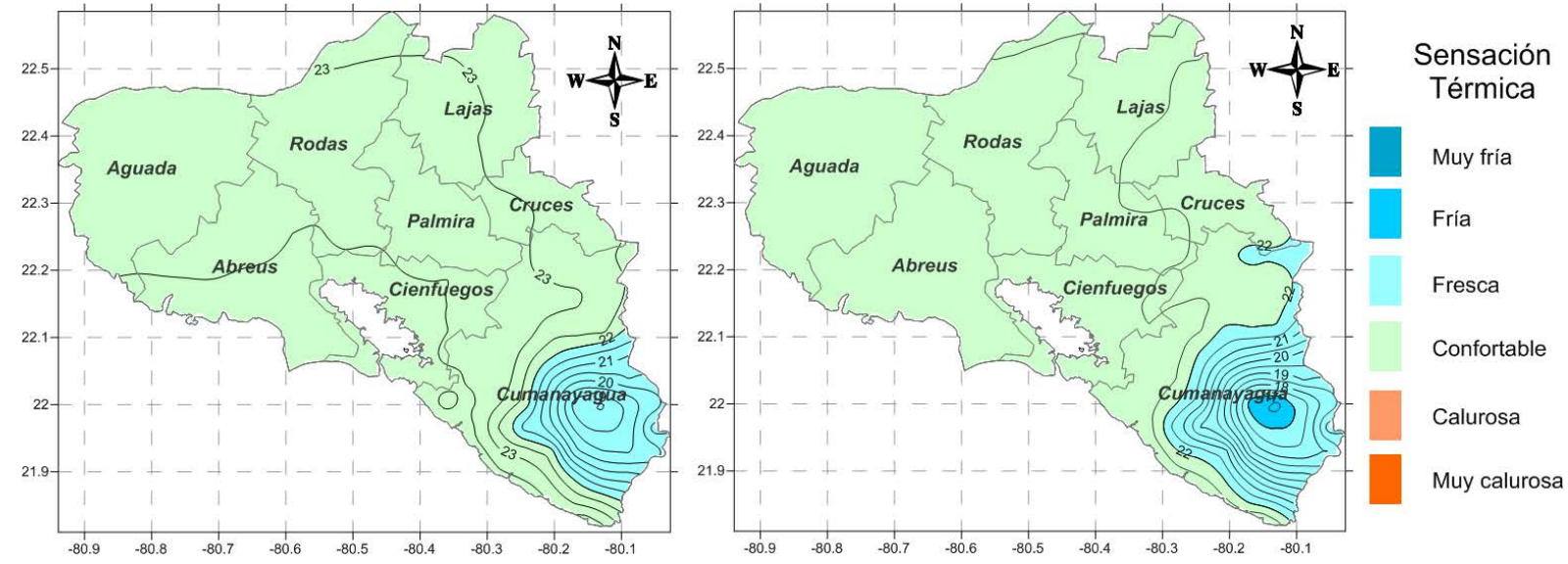

En el mes de julio a las 7:00 a.m. aunque las sensaciones térmicas adoptan configuraciones espaciales semejantes a las descritas anteriormente es necesario resaltar algunas diferencias. Según la distribución de la TE, las percepciones ligeramente frías están confinadas en alturas superiores a los $600 \mathrm{~m}$ (Figura 8, izquierda), mientras, las sensaciones frías que predominaban en las cimas más altas con influencia del viento, en este caso no son apreciables (Figura 8, derecha).

Por otra parte en el horario de la 1:00 p.m. del propio mes las sensaciones calurosas cubren la mayor parte de la provincia encontrándose fuertes gradientes hacia el municipio de Cumanayagua, sobre todo en la ladera sur del sistema montañoso donde sus niveles altos mantienen sensaciones confortables, mientras que hacia el litoral se perciben sensaciones muy calurosas (Figura 9, izquierda) las cuales desaparecen si se considera el efecto refrescante del viento en esta zona (Figura 9, derecha). Estos resultados son consistentes con lo descrito por Lecha (1993) el cual expresa que en verano solo se encuentran sensaciones de bienestar en las zonas montañosas del país. 
Figura 8. Sensación térmica del mes de julio a las 7:00 a.m. dadas por la TE (izquierda) y la TEE (derecha). Provincia Cienfuegos, período 1981-2010.
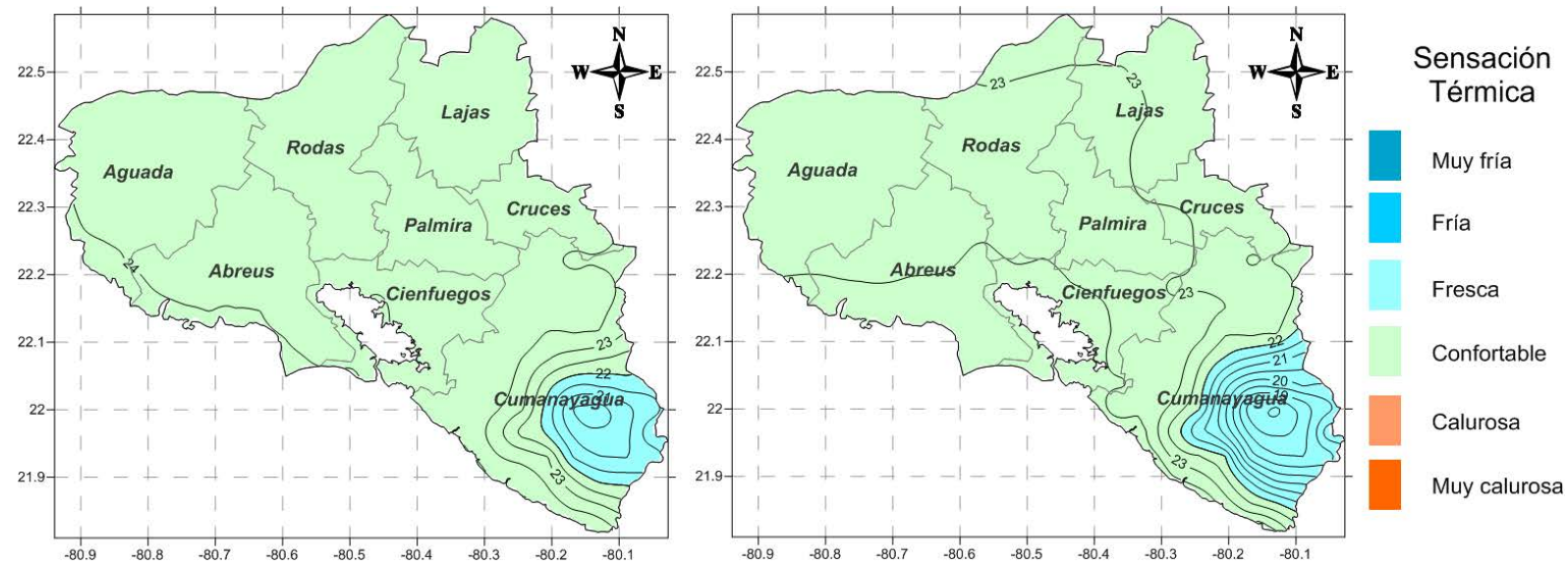

Figura 9. Sensación térmica del mes de julio a la 1:00 pm dadas por la TE (izquierda) y la TEE (derecha). Provincia Cienfuegos, período 1981-2010.
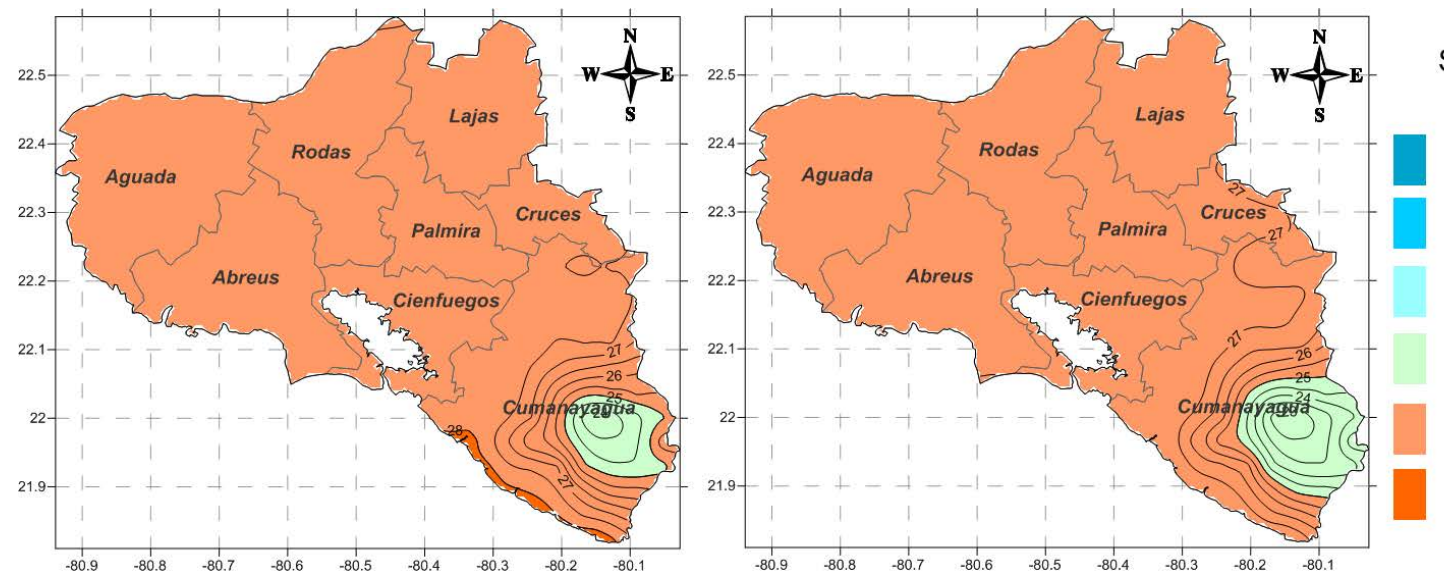

Sensación Térmica

Muy fría

Fría

Fresca

Confortable

Calurosa

Muy calurosa

\section{CONCLUSIONES}

La aplicación de los índices bioclimáticos TE y TEE permitió caracterizar temporal y espacialmente las sensaciones térmicas en la provincia Cienfuegos.

El rango diurno de las sensaciones térmicas varía en dependencia de la época del año, en consecuencia:

- Las mañanas de noviembre a abril son generalmente frescas y en menor medida frías y muy frías. En el período de mayo a octubre habitualmente son confortables (60 - 90\%) aunque también pueden ser cálidas o ligeramente frías.

- Las tardes de noviembre a marzo pueden sentirse entre calurosas, confortables y frescas. La probabilidad de las sensaciones calurosas aumenta de abril a octubre estableciéndose entre el 30 y el $70 \%$. En los meses más cálidos del año ocurren las muy calurosas alcanzando hasta un $80 \%$ como máximo.

- La zona montañosa sobresale por la permanencia de sensaciones frescas en todo el día. No obstante a las 7:00 a.m. de los meses invernales aumenta considerablemente la frecuencia de sensaciones frías y muy frías debido al efecto del viento. Las tardes de abril a octubre son confortables con alguna ocurrencia de condiciones calurosas en los meses del verano.

El análisis espacial por meses (enero y julio) y horarios (7:00 a.m. y 1:00 p.m.) permitió mostrar las características más contrastantes de las sensaciones térmicas de la provincia en función de sus particularidades físico-geográficas: 
- En las mañanas de enero la zona costera permanece con sensaciones frescas mientras que el resto de la provincia presenta sensaciones frías que al tener en cuenta la influencia del viento se extienden hacia todo el territorio excepto hacia los niveles más altos de la montaña, donde se perciben sensaciones muy frías.

- En horas de la tarde del mes de julio predominan las sensaciones calurosas en toda la zona llana de la provincia, haciéndose muy calurosas hacia el litoral sur oriental las cuales desaparecen al tener en cuenta el efecto del viento.

- Se verifica que las sensaciones confortables hacia las llanuras costeras e interiores de la provincia y frescas hacia la montaña se presentan en todos los días del año en la provincia, aunque a distinta hora del día en dependencia del mes.

- Las mayores diferencias espaciales se encontraron entre el litoral sur oriental y la zona montañosa resaltando esta última por una permanencia de sensaciones frescas o confortables y por la ausencia de sensaciones que generan estrés térmico por calor.

El efecto refrescante del viento se hace más notable hacia la zona costera en los meses de junio a septiembre debido a la entrada de las brisas marinas en horas de la tarde; mientras que de diciembre a marzo su influencia es más importante hacia la zona montañosa.

\section{REFERENCIAS}

Barcia, S. (2012). Nueva Guía Climática de la Provincia Cienfuegos. Centro Meteorológico Provincial de Cienfuegos.

Bútieva, I. V., Ilichiova, E. M., \& Kornilova, R. P. (1984). "Régimen de tiempo y sensación térmica del hombre en diferentes zonas naturales de la URSS en el período cálido del año", en Materiales de Investigaciones Meteorológicas (pp. 74-81). Moscú.

Centella, A. B., Lapinel, B., Solano, O., Vázquez, R., Fonseca, C., Cutié, V., Baez, S., Sille, J., Rosario, P., y Duarte, L. (2006). La sequía meteorológica y agrícola en la República de Cuba y la República Dominicana. (Tomo I), 174pp.

Fanger, P. O. (1972). Thermal comfort. Analysis and Applications in Environmental Engineering. Mc GrawHill. New York.

Guevara, V., Campos, A., \& León, A. (1997). Algunos elementos del potencial climático de Cuba en relación con el mejoramiento de las condiciones bioclimáticas en las edificaciones. Presented at the IV Congreso Iberoamericano sobre el Medio Ambiente., Caracas Venezuela.

Hendrick, R. L. (1959). An Outdoor Weather Comfort Index for the Summer Season in Hartford, Connecticut. (No. 40 (12)) (pp. 620-623). Bull. Amer. Meteorol. Soc.

Hougten, P., \& Yaglou, C. (1923). Determining lines of equal comfort. Heart Vent. Engrs (No. 29) (p. 163). Trans. Amer. Society.

Lecha, L. B. (1988). Los recursos climatoterapéuticos de Cuba. La Habana: Dpto. de Climatología. Instituto de Meteorología de La Academia de Ciencias de Cuba.

Lecha, L. B. (1993). Estudio bioclimático de la provincia Cienfuegos. La Habana: Editorial Academia, 131pp.

León, A. (1988). Las sensaciones de calor en el Occidente de Cuba (Trabajo de Diploma). Universidad de La Habana, Ciudad de La Habana.

Missenard, A. (1937). L'home et le climat. Paris, Francia.

Moreno, A., \& Fernández, F. (2004). "El confort climático en los entornos residenciales de las capas altas, medias y bajas de la Comunidad de Madrid: otra forma de desigualdad socioespacial2, en Distribución Espacial de la renta en la Comunidad de Madrid. Análisis y aplicaciones (pp. 153-176). España: Consejería de Economía e Innovación Tecnológica-Instituto de estadística de la Comunidad de Madrid.

Osorio, M., Esperón, R. D., \& León, A. (1991). "Meteorología y bienestar turístico en Varadero", en Revista Cubana de Meteorología, No. 4 (1).

Planos, E., Rivero, R., \& Guevara, V. (2013). "Variaciones y cambios del clima. Temperatura superficial del aire", en Impacto del Cambio Climático y Medidas de Adaptación en Cuba. Instituto de Meteorología, Agencia de Medio Ambiente, CITMA: AMA. 430 pp. 
Royé, D., Martí, E., \& Cabalar, M. (2012, September). Aproximación al comportamiento espacial del estrés térmico en Galicia mediante el uso del indice bioclimático PET. Presented at the VIII Congreso de la Asociación Española de Climatología, Salamanca, España.

Siple, P. A., \& Passel, C. S. (1945). Measurements of dry atmospheric cooling in freezing temperatures. (No. 89) (pp. 177-199). Proc. Amer. Phill. Soc.

Urriola, E. (2009). Índice de Confort, Sensación Térmica e Impacto de Olas de Calor en las Personas. Panamá: Empresa de Transmisión Eléctrica (ETESA). 